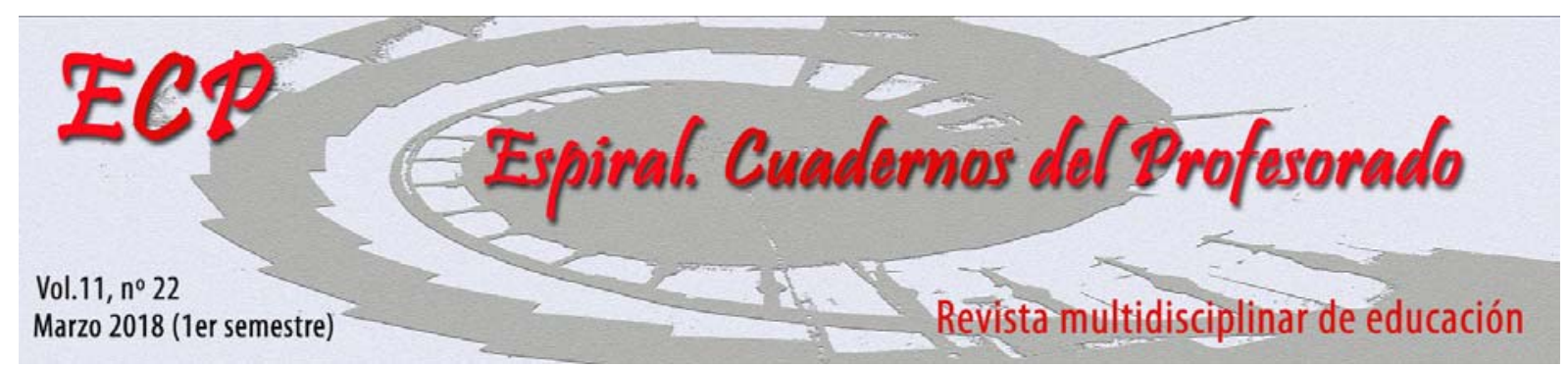

\title{
Efectos del modelo de Educación Deportiva sobre la interculturalidad: El papel del alumno-entrenador en estudiantes de primaria
}

\section{Effects of Sport Education model on interculturality: The student-coach role in elementary school students}

\author{
Federico Puente-Maxera ${ }^{(1)}$, Antonio Méndez-Giménez ${ }^{(1)}$ \\ y Diego Martínez de Ojeda Pérez ${ }^{(2)}$
}

(1) Universidad de Oviedo, España

(2) CEIP Feliciano Sánchez Saura de Cartagena, Murcia, España

\begin{abstract}
RESUMEN: El presente estudio ha tenido un doble propósito: (a) examinar el impacto de una unidad didáctica de ultimate bajo las premisas del Modelo de Educación Deportiva (MED) sobre el comportamiento y la sensibilidad interculturales de estudiantes de sexto de primaria en un grupo de alta diversidad cultural; y (b) conocer su incidencia específica en el rol de alumno-entrenador. Participaron 18 estudiantes (en su totalidad de sexo masculino) con edades comprendidas entre los 11 y 13 años $(M=11.65 ; D T=.76)$ pertenecientes a un colegio público ubicado en el sur de España. Se llevó a cabo un diseño cuasiexperimental con medidas pretest y postest. La información cuantitativa fue recabada a través de dos cuestionarios para estudiantes, mientras que los datos cualitativos se recogieron mediante un diario docente y entrevistas tanto al profesorado como al alumnado. Los resultados más destacados parecen haber revelado, por un lado, el potencial del MED para favorecer la integración de los estudiantes independientemente de sus características individuales y, por otro, el uso de la pizarra como eficaz herramienta de andamiaje respecto al rol de alumno-entrenador. Por su parte, la intervención no provocó diferencias significativas en el comportamiento intercultural de los participantes.
\end{abstract}

Palabras clave: Educación física, modelos de enseñanza, educación deportiva, educación intercultural, sensibilidad intercultural.

ABSTRACT: This study had two main goals: the first was (a) to analyze the impact of an ultimate's Sport Education model-based unit on the sixth-grade students' intercultural sensitivity and behavior in multicultural contexts; and the second was (b) to know the specific influence on the student-coach role and its consequences on intercultural perspectives. Eighteen male students aged from 11 to $13(M=11.65 ; D T=.76)$ from a southern Spain state school took part in this study. A quasi-experimental design, pretest and postest measures, was carried out. Data collection included two student questionnaires, a teacher's diary and interviews for both students and teacher. Results suggest that SEM-based interventions had shown the potential to promote integration among students beyond their individualities. Furthermore, the use of small whiteboards has been showed as positive strategy for scaffolding the student-coach role. On the other hand, the intervention has no promoted significant differences on students' intercultural behavior.

Key words: Physical education, instruction models, sport education, intercultural education, intercultural sensitivity.

Puente-Maxera, F., Méndez-Giménez, A., y Martínez de Ojeda, D. (2018). Efectos del modelo de Educación Deportiva sobre la interculturalidad: El papel del alumno-entrenador en estudiantes de primaria. Espiral. Cuadernos del Profesorado, 11(22), 1-14.

Fecha de recepción: 23/04/2017

Fecha de aceptación: 20/09/2017
Correspondencia: fedepuentem@hotmail.com (F. Puente-Maxera) 


\section{Introducción}

Uno de los componentes pedagógicos que más ha evolucionado a lo largo del tiempo ha sido la centralidad del proceso de enseñanza-aprendizaje, desde enfoques centrados en la figura docente hacia otros centrados en el estudiante (Weimer, 2002). Así pues, la estructura jerárquica de verticalidad da paso a un esquema horizontal, los esquemas de comunicación multidireccional sustituyen a aquellos unidireccionales (Johnson y Miller, 1993) y, en definitiva, se busca que el alumnado se convierta en el verdadero protagonista de su aprendizaje. De esta forma, el docente, asume las labores de facilitar y orientar el proceso de enseñanza-aprendizaje (Sierra-Arizmendiarrieta, Méndez-Giménez, y Mañana-Rodríguez, 2013).

En el ámbito de la Educación Física (EF) son varios los modelos pedagógicos que comparten estas características (Metzler, 2005). Uno de ellos es el MED, de gran repercusión en el panorama nacional e internacional. Las diferentes revisiones que incluyen los estudios que analizan aquellos efectos que produce este modelo de enseñanza así lo constatan (véase Hastie, Martínez de Ojeda, y Calderón, 2011; Wallhead y O'Sullivan, 2005; Evangelio, González-Víllora, Serra-Olivares, y PastorVicedo, 2016). El MED nace con el propósito de brindar a los practicantes de la EF escolar experiencias deportivas auténticas y significativas (García-López \& Gutierrez, 2016; Siedentop, Hastie, y Van der Mars, 2011). Se compone de una serie de elementos básicos que lo caracterizan: (a) la afiliación, (b) las temporadas, (c) el registro de datos, (d) la competición formal, (e) la festividad, y (f) el evento final. Numerosos estudios han constatado las bondades de cada uno de dichos elementos, como es la oportunidad para el desarrollo social promovida por la pertenencia prolongada a un mismo equipo durante toda una unidad didáctica (García-López y Gutiérrez, 2016). Igualmente, los estudiantes, como miembros fijos de un equipo determinado, desempeñan una serie de responsabilidades de diversa índole (Evangelio et al. 2016), entre otras, entrenador, preparador físico, encargado de material, etc.

Estudios realizados desde los postulados del MED han puesto de manifiesto que uno de los roles que comporta mayor liderazgo y, a su vez, mayor responsabilidad, es el de alumno-entrenador (Hastie y Sinelnikov, 2006). En torno a este, varias investigaciones han ido dirigidas a conocer los aspectos más determinantes en su abordaje y aprendizaje. En un estudio con adolescentes, Wallhead y O'Sullivan (2007) llevaron a cabo un microanálisis sobre la participación de un estudiante al actuar como entrenador respecto a sus compañeros de equipo. Como aspectos positivos evidenciaron, por un lado, la utilidad de la enseñanza recíproca en la adquisición de conocimiento y cultura deportiva, y, por otro, el uso de tarjetas con actividades (task cards) como eficaz proceso de andamiaje. Con respecto a este último, y por aproximarnos al concepto de andamiaje, consideramos oportunas las aportaciones de Wood, Bruner y Ross (1976, citado por Lin, T.C. et al., 2012) quienes lo definen como el soporte pedagógico que un adulto o un igual más experto proporciona a un aprendiz para que pueda completar una tarea que está por encima de su capacidad inicial. Se trata, así pues, de un mecanismo orientado a favorecer el desarrollo de la capacidad autorreguladora de los estudiantes (López y Hederich, 2010). Posteriormente al trabajo de Wallhead y O'Sullivan (2007), en un estudio prolongado con 26 adolescentes, Farias, Hastie, y Mesquita (2017) pudieron dilucidar ciertos aspectos relevantes en el rol de alumno-entrenador. En su caso, emplearon procesos de andamiaje muy variados (conocimiento previo del alumnado, reflexión sobre la práctica, etc.) y evidenciaron altos beneficios respecto a determinadas estrategias de enseñanza, siendo el descubrimiento guiado una de estas. Dicha estrategia propició situaciones de reflexión-acción confirmadas previamente como fundamentales a la hora de implicar al alumnado en su aprendizaje (Dyson, Griffin y Hastie, 2004; Ward y Lee, 2005). Los efectos más sobresalientes en el estudio de Farias et al. (2017) se reflejaron en la capacidad de los alumnos-entrenadores para identificar problemas tácticos emergentes y para desarrollar habilidades comunicativas.

En los estudios mencionados se emplearon distintos elementos dirigidos a facilitar los procesos de comunicación. En el caso de Wallhead y O'Sullivan (2007) se utilizaron las mencionadas "task cards", mientras que en Farias et al. (2017), además de utilizar estas últimas, se emplearon otros recursos como, por ejemplo, un manual que incluyó juegos, dibujos y reglas. Acorde con ello, el 
presente estudio pretende abordar un planteamiento similar a partir de la inclusión de pequeñas pizarras.

No obstante, las mejoras destacadas en los dos estudios previos referidos no son igualmente alcanzables de forma genérica por todo el alumnado. Farias et al. (2017) sostienen que los procesos de andamiaje son dinámicos y, como tales, responden a las características individuales de los estudiantes. En ese sentido, un rasgo distintivo, ampliamente analizado por la literatura, ha sido el país de procedencia del alumnado. La presencia de estudiantes de dichas características ha propiciado el origen de un nuevo enfoque, la educación intercultural. Lejos de ser un campo de estudio exclusivo, ajeno a la educación formal y con un currículum en paralelo (Besalú, 2002), la educación intercultural viene entendida por una amplia mayoría de autores expertos en la materia como un enfoque o una mirada diferente de una educación para todos (Aguado y Ballesteros, 2015; García y Goenechea, 2009). Estudios realizados desde esta óptica han confirmado el potencial de los programas en clave intercultural para incidir en distintas dimensiones competenciales del aprendizaje. Un ejemplo de ello se observa en la sensibilidad intercultural, enmarcada en la dimensión afectiva de la comunicación intercultural, y que ha sido estudiada en distintas etapas académicas, tanto en primaria (Sanhueza y Cardona, 2009) y secundaria (Ruokonen y Kairavuori, 2012) como a nivel universitario (Arslan, Günçavdi, y Polat, 2014).

No obstante, en el área de la EF existe cierta escasez sobre estudios desarrollados desde una perspectiva intercultural (Derri, Kellis, Vernadakis, Albanidis, y Kioumourtzoglou, 2014; Kouli y Papaioannou, 2009). Aún más difícil supone encontrar propuestas que se posicionen desde modelos pedagógicos centrados en el alumnado. Del mismo modo, los propósitos que parecen haber guiado estas investigaciones han tenido un marcado tinte compensatorio, en muchos casos, y folclórico, en otros, quedando encuadrados estos últimos en lo que desde Francia se ha convenido en llamar "pedagogía del cuscús" (Jordán, 1999) y que alude a la incorporación a nivel escolar de una serie de aspectos exóticos propios de las culturas presentes en el aula (p.ej., gastronomía o vestimenta) y que son tratados de manera puntual, sin llegar a ser incluidos en el currículum ordinario (Jiménez y Goenechea, 2014).

En virtud de lo expuesto, el presente estudio persigue un doble propósito. Por un lado, (a) examinar el impacto de una unidad didáctica de ultimate bajo las premisas del MED sobre el comportamiento y la sensibilidad interculturales de 18 estudiantes de sexto de primaria en un grupo de alta diversidad cultural; y por otro, (b) conocer su incidencia específica en el rol de alumnoentrenador, así como su repercusión en clave intercultural.

\section{Material y método}

\section{Enfoque}

Se empleó una metodología mixta (cuantitativa y cualitativa) y se utilizó un diseño cuasiexperimental (Campbell y Stanley, 1963) con medidas pretest y postest. Estudios previos basados en el MED, tanto en el panorama nacional como internacional, han seguido este tipo de diseños (Calderón, Martínez de Ojeda, Valverde, y Méndez-Giménez, 2016; Cuevas, García-López, y Contreras, 2015; Hastie, Buchanan, Wadsworth, y Sluder, 2009). Por su parte, el estudio asume una aproximación metodológica de carácter mixto, empleando tanto técnicas de recogida cuantitativas como cualitativas, tal y como ya lo abordaran estudios MED anteriores (Gutiérrez, García-López, Chaparro y Fernández, 2014; Gutiérrez, García-López, Hastie, y Calderón, 2013; Méndez-Giménez, Puente-Maxera, y Martínez de Ojeda, 2017).

\section{Participantes}

El estudio contó con la participación de 18 estudiantes de sexto curso de primaria, con edades comprendidas entre los 11 y 13 años $(M=11.65 ; D T=.76)$, pertenecientes a un colegio público ubicado en la Región de Murcia. En cuanto al género, la totalidad de los participantes $(N=18)$ fueron de sexo masculino. El grupo fue seleccionado por su disponibilidad y alto grado de diversidad cultural (mayormente, alumnado de origen marroquí) alcanzando un porcentaje del $50 \%$. Por su parte, el 
maestro encargado en impartir de docencia contaba con 11 años de experiencia y 4 implementando el MED. El estudio contó con el consentimiento del centro educativo, así como de los padres y madres del alumnado.

\section{Procedimiento}

Se llevó a cabo una unidad didáctica de ultimate bajo las premisas del MED, de diez sesiones de 60 minutos de duración. El grupo-clase fue organizado a partir de 4 equipos heterogéneos en cuanto a nacionalidad y nivel de aprendizaje. Las responsabilidades asumidas en cada uno de los equipos fueron las siguientes: entrenador, preparador físico, encargado de material y responsable de riesgos laborales. En aquellos grupos donde existían más de cuatros integrantes, se repitió el rol de encargado de material. Del mismo modo, los equipos realizaron la labor de árbitros y anotadores (duty team) sirviéndose de una hoja de registro previamente confeccionada (véase Tabla 1). Entre otros aspectos (e.g., goles conseguidos), esta ficha incluía un checklist de deportividad compuesto por diez ítems (e.g., respeta las decisiones arbitrales).

Siguiendo los postulados del MED, la intervención progresó a partir de las siguientes fases: (a) introductoria, donde se dio a conocer el contenido, se organizaron los grupos y se repartieron los roles; (b) dirigida, en la que las actividades fueron impartidas por el maestro introduciendo andamiajes parciales hacia la figura del alumno-entrenador, (c) de práctica autónoma, donde los equipos tomaban sus propias decisiones y resolvían distintos problemas; (d) de competición formal, en la que se alternaban períodos de práctica con partidos de competición; y (e) el evento final, en el que los alumnos fueron partícipes de una actividad de síntesis dirigida a destacar y premiar sus actuaciones a lo largo de la unidad. La Tabla 2 resume los contenidos de cada sesión en cada fase de la temporada de ultimate.

Tabla 1.

Hoja de anotación.

\begin{tabular}{l}
\hline NOMBRE DEL ANOTADOR/A: \\
TIPO DE PARTIDO: Amistoso $\square$ Competición \\
EQUIPO: \\
\hline GOLES CONSEGUIDOS: \\
1-2-3-4-5-6-7-8-9-10-11-12-13-14-15-16-17-18-19-20 \\
\hline JUEGO LIMPIO (cada uno vale 1 punto): \\
\hline Saluda al equipo contrario al inicio del partido. \\
Respeta las decisiones arbitrales. \\
Respeta en todo momento el material. \\
Respeta a los compañeros durante todo el encuentro (habla sin gritar ni insultar). \\
Respeta a los contrarios durante todo el encuentro. \\
Anima a los compañeros de equipo \\
Se esfuerzan durante todo el encuentro \\
Se ponen de acuerdo rápido sin discutir (en cambios, en quién saca,...) \\
Participan de forma equitativa (no hay alumnos que siempre sean los que lanzan) \\
Saluda al equipo contrario al final del partido.
\end{tabular}

Asimismo, se desarrolló un protocolo de formación específica para el rol de alumnoentrenador a lo largo de 3 sesiones realizadas en los tiempos de recreo. En dicho protocolo se abordó la enseñanza del uso de la pizarra como herramienta de comunicación, siendo tratados distintas 
estrategias de comunicación y mecanismos de feedback (véase Figura 1). Otra novedad propia de la intervención estribó en el uso de materiales autoconstruidos (frisbees) en sesiones de contingencia previas a la unidad.

Tabla 2.

Contenidos de las sesiones en cada fase de la temporada de ultimate.

\begin{tabular}{|c|c|}
\hline \multicolumn{2}{|r|}{ UD ULTIMATE } \\
\hline SESIONES & Contenido \\
\hline 1. (I) & Explicación, formación de equipos, reparto de roles. \\
\hline 2. (D) & $\begin{array}{l}\text { Juegos de pase y recepción de forma estática y dinámica. Juegos reducidos en superioridad } \\
\text { numérica en ataque } 2 \mathrm{x} 0 ; 3 \mathrm{x} 0 \text {. }\end{array}$ \\
\hline 3. (D) & $\begin{array}{l}\text { Juegos de repaso. Jugadas combinadas. Iniciación al desmarque. Juegos en superioridad } \\
\text { numérica en ataque } 3 \times 1 ; 2 \times 1 \text { (situación para atacar; cómo se defiende en inferioridad } \\
\text { numérica) }\end{array}$ \\
\hline 4. (PR) & $\begin{array}{l}\text { Alumno entrenador. Rapidez de transición del disco. Juegos reducidos de repaso en } \\
\text { superioridad numérica. Iniciación al desmarque. Juegos en igualdad numérica } 2 \times 2 \text { con } \\
\text { agarre en la zona. }\end{array}$ \\
\hline 5. (PR) & $\begin{array}{l}\text { Práctica autónoma. Explicación de la hoja de anotación. Partidos de práctica con duty team } \\
\text { e incidiendo en algún aspecto táctico (p.ej., todos los atacantes deben estar en campo } \\
\text { contrario; defensa } 1 \times 1 \text { ). }\end{array}$ \\
\hline 6. (PR) & $\begin{array}{l}\text { Práctica autónoma. Explicación de la hoja de anotación. Partidos de práctica con duty team } \\
\text { e incidiendo en algún aspecto táctico. }\end{array}$ \\
\hline 7. (PR) & Práctica autónoma. Partidos de práctica con duty team. \\
\hline 8. (CF) & Competición regular, formato liga (3 partidos) \\
\hline 9. (CF) & Competición regular, formato liga (3 partidos) \\
\hline 10. (EC) & Partidos finales de exhibición. Entrega de diplomas. \\
\hline
\end{tabular}

Nota: Fases I: Introductoria; D: Dirigida; PR: Práctica; CF: Competición formal; EC: Evento culminante

\section{Instrumentos}

\section{Percepción de los estudiantes}

Respuesta emocional positiva. Se hizo uso de la sub-escala Respuesta Emocional Positiva (REP), correspondiente a la Escala de Sensibilidad Intercultural desarrollada por Chen y Starosta (2000) y adaptada por Sanhueza y Cardona (2008). La sub-escala está compuesta por 7 ítems (e.g., "Me gusta relacionarme con compañeros/as de otros países) valorados a partir de una escala Likert que oscila entre 1 (Nunca es cierto) a 5 (Siempre es cierto). Sanhueza (2010) obtuvo un alfa de Cronbach de $0.75(N=389)$ para toda la escala en su conjunto. Una puntuación alta indicaría que el sujeto posee una buena sensibilidad intercultural, caracterizada por respuestas positivas hacia sus compañeros culturalmente diferentes. Los estudiantes asignaron dos valores para cada ítem, expresando su opinión respecto a dos momentos: antes (pretest) y después (postest) de la unidad.

Comportamiento intercultural. Se aplicó la Escala de Autoevaluación del Comportamiento del Estudiante (Kellis, Vernadakis, Albanidis, Derri, y Kourtesses, 2010). Este instrumento mide el desarrollo moral y social de los estudiantes en contextos multiculturales. Su versión original consta de 18 ítems relacionados con cinco habilidades sociales: irresponsabilidad; recompensa; ayuda; familiaridad/relaciones; $y$ metas (véase la Tabla 3). Se realizó una traducción-retrotraducción de los ítems de la versión de esta herramienta siguiendo las recomendaciones de Hambleton, Merenda, y Spielberger (2005). Las adaptaciones realizadas acarrearon modificaciones en la organización de los 
ítems y en la terminología (cambios idiomáticos al traducir de la lengua inglesa al castellano: e.g., la frase "talk to others offensively..." fue sustituida en castellano por "digo cosas negativas a otros..."). El instrumento utilizó una escala tipo Likert de 1 "nunca" a 5 "siempre". El alfa de Cronbach obtenida en cada una de las subescalas en el estudio de Kellis et al. (2010) fue, respectivamente, $\alpha=$ .78 (metas); $\alpha=.88$ (relaciones); $\alpha=.76$ (recompensa); $\alpha=.71$ (ayuda); y .73 (irresponsabilidad). La dimensión "metas" fue descartada al no reunir aspectos de interés para el presente estudio.

Entrevistas. Una vez finalizada la intervención, los estudiantes fueron entrevistados dispuestos en grupos de cuatro-cinco (coincidiendo con los equipos establecidos en la unidad), tal y como recomiendan Ennis y Chen (2012). Se registraron un total de cuatro entrevistas semi-estructuradas en las que los participantes respondieron a preguntas acerca de su experiencia a lo largo de la unidad. Un grupo de expertos revisó y ultimó los guiones de las entrevistas.

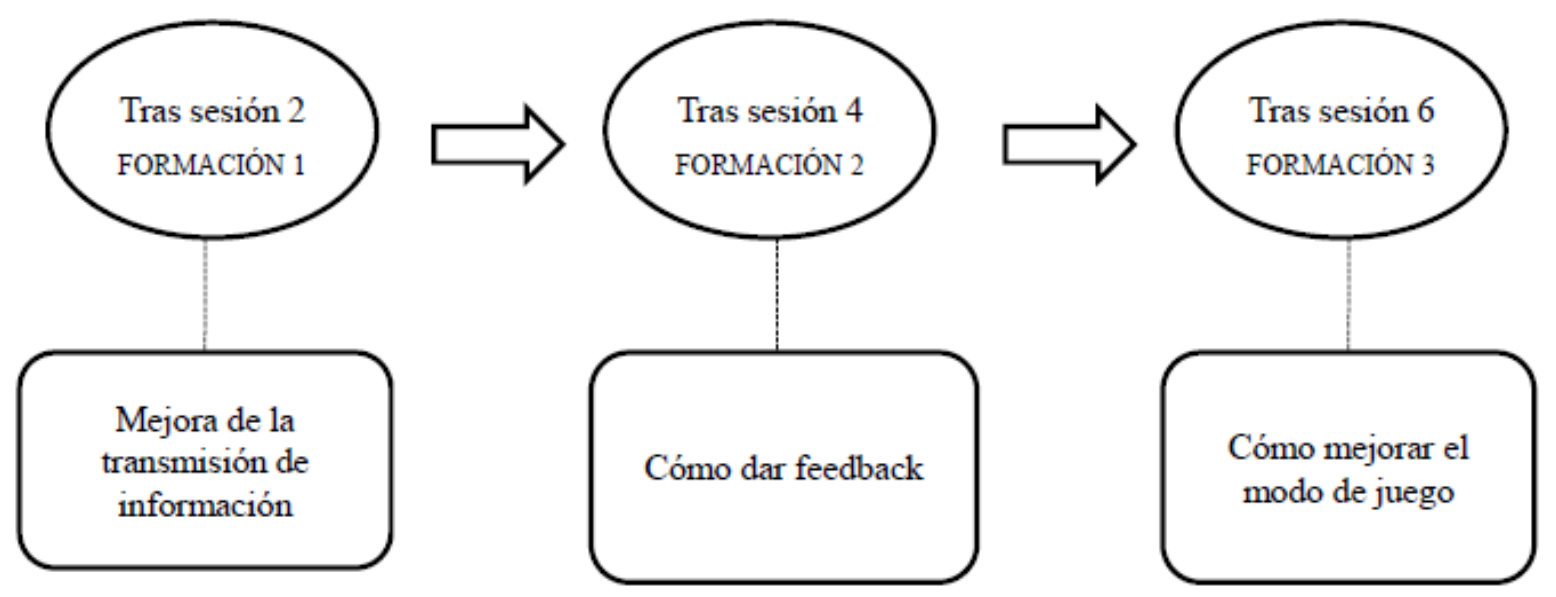

Figura 1. Protocolo de formación del alumno-entrenador y temporalización.

\section{Percepción del docente}

Diarios docentes. El maestro responsable de impartir docencia registró sus observaciones e incidencias más relevantes de cada sesión (Erickson, 1989). El diario empleado en este estudio presenta una estructura de tres ejes temáticos derivados de una serie de categorías emergentes.

Entrevistas. Se realizaron tres entrevistas semi-estructuradas (Cohen y Manion, 2002) atendiendo a distintas fases de la intervención (antes, durante y después de la misma). Se abordaron temas relacionados con la interculturalidad y el proceso de enseñanza-aprendizaje. Todas las entrevistas fueron transcritas para su posterior análisis.

\section{Análisis de datos cuantitativos}

Los datos cuantitativos fueron analizados utilizando el paquete estadístico SPSS (versión 24.0). Respecto a la fiabilidad de la REP, se obtuvieron alfas de Cronbach de .92 y .90 en pretest y postest, respectivamente, mientras que en el comportamiento intercultural se obtuvieron alfas de $.75 \mathrm{y}$ .69 , respectivamente (véase la Tabla 4). Estos valores excedían el criterio de .70 establecido por la literatura (Kline, 1998), salvo en el postest del comportamiento intercultural $(\alpha=.69)$. No obstante, se mantuvieron los análisis subsiguientes considerando la franja de edad del alumnado y el gran interés que suponía para el estudio. El resto de variables fueron desestimadas por no obtener índices de fiabilidad aceptables. Por su parte, se solicitó la prueba de Shapiro-Wilk para valorar la normalidad de las variables cuantitativas, obteniéndose valores de Sig. $<.05$. Por tanto, en los análisis subsiguientes se emplearon pruebas no paramétricas. Para comparar las puntuaciones de cada grupo a través del tiempo se empleó la prueba de los rangos con signo de Wilcoxon de medidas relacionadas introduciendo las medidas del pretest y post-test. 
Tabla 3.

Escala de Autoevaluación del Comportamiento del Estudiante (adaptado de Kellis et al. 2010).

\begin{tabular}{rllllll}
\hline 1. & Me gusta tener amigos/as de otros países/culturas. & 1 & 2 & 3 & 4 & 5 \\
\hline 2. & Digo cosas negativas a niños/as de otros países sobre el lugar de donde vienen. & 1 & 2 & 3 & 4 & 5 \\
\hline 3. & $\begin{array}{l}\text { Felicito a los compañeros/as de otros países/culturas que se esfuerzan en hacer los } \\
\text { ejercicios. }\end{array}$ & 1 & 2 & 3 & 4 & 5 \\
\hline 4. & $\begin{array}{l}\text { Acepto la ayuda de compañeros/as de otros países cuando no puedo realizar un } \\
\text { ejercicio. }\end{array}$ & 1 & 2 & 3 & 4 & 5 \\
\hline 5. & Me gusta jugar con niños/as de otros países/culturas. & 1 & 2 & 3 & 4 & 5 \\
\hline 6. & $\begin{array}{l}\text { Hago comentarios negativos sobre el aspecto físico de mis compañeros/as de otros } \\
\text { países/culturas. }\end{array}$ & 1 & 2 & 3 & 4 & 5 \\
\hline 7. & $\begin{array}{l}\text { Doy ánimos a mis compañeros/as de otros países/culturas cuando tienen } \\
\text { dificultades con los ejercicios. }\end{array}$ & 1 & 2 & 3 & 4 & 5 \\
\hline 8. & $\begin{array}{l}\text { Me preocupo por que mis compañeros/as de otros países estén seguros cuando } \\
\text { hacen los ejercicios. }\end{array}$ & 1 & 2 & 3 & 4 & 5 \\
\hline 9. & Tengo amigos/as de otros países/culturas. & 1 & 2 & 3 & 4 & 5 \\
\hline 10. & Insulto a mis compañeros/as de clase de otros países/culturas sin motivo alguno. \\
\hline 11. & $\begin{array}{l}\text { Felicito a los jugadores oponentes de otros países/culturas sin importar el resultado } \\
\text { del juego. }\end{array}$ & 1 & 2 & 3 & 4 & 5 \\
\hline 12. & Pido ayuda a compañeros/as de otros países/culturas para mejorar en un ejercicio. & 2 & 3 & 4 & 5 \\
\hline 13. & Felicito a mis compañeros/as de otros países/culturas cuando juegan limpio. & 1 & 3 & 4 & 5 \\
\hline 14. & $\begin{array}{l}\text { Ayudo a mis compañeros/as de otros países/culturas cuando no consiguen realizar } \\
\text { un ejercicio. }\end{array}$ & 1 & 2 & 3 & 4 & 5 \\
\hline
\end{tabular}

\section{Análisis de datos cualitativos}

La información cualitativa fue analizada de forma manual a partir de técnicas inductivas (síntesis, codificación y comparación de respuestas) y siguiendo las fases de reducción, exposición de la información, así como de extracción de conclusiones (Massot, Dorio, y Sabariego, 2004). Las transcripciones de las entrevistas fueron leídas y releídas. Posteriormente, los distintos constructos orientaron el análisis de datos, agrupando y codificando la información por niveles de especificidad. Finalmente, los datos fueron fragmentados en una serie de ideas codificadas y clasificadas en dos categorías: (1) El rol de alumno-entrenador y su repercusión en la comunicación intercultural, y (2) Relaciones sociales e interculturalidad.

La validez interpretativa fue consolidada mediante técnicas de triangulación metodológica (Boyd, 2001) siendo combinados los paradigmas cuantitativo (e.g., uso de cuestionarios) y cualitativo (e.g., realización de entrevistas). Estas estrategias permitieron conocer con mayor profusión las variables objeto de estudio. Así pues, se identificaron aquellas similitudes y discrepancias más destacadas entrando en un estado de permanente retroalimentación. 
Tabla 5 .

Estadísticos descriptivos de la REP y Comportamiento intercultural en la muestra total y procedencia.

\begin{tabular}{lccccc}
\hline & \multicolumn{2}{c}{ Pretest } & \multicolumn{2}{c}{ Postest } & \\
\hline & $M$ & $D T$ & $M$ & $D T$ & $r$ \\
\hline REP & & & & & \\
\hline N total & 4.35 & 0.92 & $4.57^{*}$ & 0.62 & 0.20 \\
Autóctonos & 4.23 & 1.16 & 4.42 & 0.76 & \\
Inmigrantes & 4.46 & 0.69 & 4.71 & 0.46 & \\
\hline Comportamiento & & & & & \\
Intercultural & & & & & 0.31 \\
\hline N total & 3.86 & 0.55 & 3.97 & 0.47 & \\
Autóctonos & 3.88 & 0.66 & 3.97 & 0.56 & \\
Inmigrantes & 3.84 & 0.47 & 3.96 & 0.40 & \\
\hline
\end{tabular}

Nota: ${ }^{*} p<.05 ; M=$ Media; DT = Desviación típica; $R E P=$ Respuesta Emocional Positiva; $r$ de Cohen $(1988)=$ tamaño del efecto para pruebas no paramétricas.

\section{Resultados}

\section{Resultados cuantitativos}

La Tabla 5 recoge los estadísticos descriptivos en las variables REP y Comportamiento Intercultural para la muestra total y en función de la procedencia de los estudiantes (ver figuras y tablas). Se presentan, a su vez, los valores obtenidos en los análisis del tamaño del efecto con la $N$ total (Fritz, Morris, y Richler, 2012).

Atendiendo a los participantes del estudio, en su totalidad, se evidenciaron mejoras significativas $(p<.05)$ en la REP sin manifestarse cambios por razones de procedencia. Por su parte, en la variable Comportamiento Intercultural no se observaron cambios significativos en ninguna de sus condiciones. Respecto al tamaño del efecto, Cohen (1988) informó de los siguientes intervalos para $r$ : de .1 a .3 : efecto pequeño; de .3 a .5 : efecto intermedio; y .5 y superior: efecto fuerte.

Finalmente, la Tabla 6 sintetiza los resultados de las comparaciones intragrupo mediante la prueba rangos de Wilcoxon (ver figuras y tablas).

\section{Resultados cualitativos}

A continuación, se exponen los resultados cualitativos derivados de los instrumentos de recogida y distribuidos en las dos categorías resultantes:

(I) El rol de alumno-entrenador y su repercusión en la comunicación intercultural.

El docente, antes del inicio de la experiencia, esperaba un buen dominio del rol de alumnoentrenador por parte de aquellos estudiantes implicados en la labor. Aseguró que la competencia lingüística inicial era suficiente en la mayoría del alumnado, lo que le llevó a no prever dificultades importantes en el proceso de comunicación: "El entrenador que no sepa comunicarse tendrá problemas, pero no significativos". En esta misma línea, una vez concluida la intervención, en clave intercultural, el maestro no evidenció ningún tipo de carencia comunicativa. Sin embargo, de los comentarios de los estudiantes, sí se observaron ligeros conflictos comunicativos a nivel intragrupal: "Había veces que hablaban en marroquí y no nos esterábamos", comentó un estudiante. No obstante, los propios estudiantes aseguraron haber resuelto estas situaciones en el transcurso de la unidad, justificando la afiliación y la responsabilidad individual como razones principales. 
Exceptuando estos casos específicos, la interculturalidad per se no fue expuesta como variable decisiva en el rol de alumno-entrenador. Según la percepción docente, la eficacia en la transmisión de la información $\mathrm{y}$, en consecuencia, en el aprendizaje pareció ser explicada a partir de dos factores: por un lado, la personalidad del alumno-entrenador en cuestión $\mathrm{y}$, por otro, la inclusión de la pizarra como herramienta comunicativa. Respecto al primero, el maestro destacó una serie de características que debía reunir un alumno-entrenador, entre otras, la iniciativa, el liderazgo y el carisma. El docente observó que, en muchos grupos, el rol de alumno-entrenador había sido designado de forma democrática y considerando, a su vez, la idoneidad de sus miembros para ejercer como tal. Así fue confirmado por un estudiante: "Hemos decidido que el entrenador fuese [alumno] porque explica muy bien las cosas".

Por otro lado, el maestro confirió especial importancia a la inclusión de la pizarra reconociéndola como causante de mejoras tanto en el aprendizaje deportivo como en la comunicación no verbal: "Es mucho más visual. Facilita la comprensión”, aseguró el maestro. Los comentarios de los estudiantes apuntaron en esa dirección avalando, de forma unánime, la inclusión de la pizarra en futuras intervenciones. Quienes ejercieron el rol de alumno-entrenador aseguraron que esta herramienta les facilitó su labor: "Ayudaba mucho porque ellos lo sabian, en verdad, pero no lo entendían. Entonces, cogía la pizarra, se lo explicaba asi y se daban cuenta. [...] Me ha ayudado a explicar", comentó un estudiante. "Me ha gustado más", sostuvo otro alumno también en calidad de entrenador. Por su parte, el resto de miembros de cada equipo coincidieron en reconocer a la pizarra como un material útil para clarificar la información, así como para comprender mejor las tareas encomendadas: "Ha sido mejor porque con la pizarra lo entendemos más fácil", comentó un alumno. Muchos estudiantes, entrenadores o no, destacaron la utilidad de la pizarra a la hora de crear estrategias y jugadas propias. El docente, asimismo, comprobó que los conceptos de táctica y estrategia emergieron con mayor claridad y frecuencia respecto a unidades anteriores.

Sin embargo, en un intento por converger estos dos factores ilustrados, el docente observó que no todos los estudiantes reunían los requisitos para utilizar la pizarra de manera eficaz: "El tema de la pizarra tiene su grado de complejidad. [...] Si me preguntas por el modelo, sin pizarra, creo que cualquier niño puede actuar de entrenador". Aseguró que, si bien cualquier alumno podía ejercer de puro transmisor, el uso de la pizarra para plasmar ideas propias, crear información y dar feedback exigía mayores cualidades. Sobre ello, destacó la importancia del protocolo de formación, siendo fundamental para utilizar la pizarra de forma más autónoma y explicar con mayor precisión: "Las sesiones de formación les están viniendo muy bien. [...] Las pizarras empiezan a ser una herramienta con un peso significativo en sus explicaciones". Finalmente, el docente destacó la iniciativa y el interés del alumnado a la hora de trabajar con este recurso, incluso, en momentos no previstos para su utilización, como fue el caso de los entrenamientos. Un alumno lo explicó de la siguiente manera: "Durante el entrenamiento, planeábamos (jugadas) mientras otros practicaban el pase".

Los frisbees autoconstruidos constituyeron otro recurso destacado por los participantes. $\mathrm{Su}$ pertinencia en esta experiencia fue apoyada por unanimidad entre los estudiantes. Entre otras razones, subrayaron la posibilidad de personalizar el material y hacerlo más atractivo y manejable: "Lo hemos hecho a nuestro estilo porque es lo que creemos que nos gusta más", dijo un estudiante. "El nuestro 
es más rápido", sostuvo otro estudiante contrastándolo con el material convencional. Una comparación similar, aunque en términos de motivación, fue esbozada por el docente: "Trabajar con su propio material, hecho por ellos, siempre es más motivante que trabajar con el material tradicional", comentó. Si bien el alumnado, de manera unánime, aprobó su inclusión para futuras intervenciones, algunos matizaron que su uso debería estar sujeto al contenido trabajado: "Habrá cosas que sí y otras que no", comentó un estudiante; "Para las indiacas estaría bien", señaló un compañero. El maestro compartió ese parecer: "En una unidad de fútbol sala, no sé si estarían tan contentos construyendo sus balones y jugando ellos. Pero en una unidad como puede ser el ultimate o indiacas, o alguno que sea con un material más alternativo, seguro que sí”. Por último, cabe destacar cómo emergieron las nociones de valoración y respeto en algunos grupos. Al ser preguntados sobre el disfrute de este material, un alumno comentó: "Sí, [ha gustado] porque así podemos respetar nuestro trabajo".

(II) Relaciones sociales e interculturalidad.

El maestro anticipó con acierto que las relaciones sociales acaecidas serían muy positivas tanto a nivel intra como intergrupal. Entre las decisiones metodológicas más destacadas, reconoció la importancia de la afiliación, la asignación de roles y los agrupamientos heterogéneos. Respecto a estos últimos, el origen étnico-cultural fue identificado, entre otras, como condición de dicha heterogeneidad. No obstante, el docente le confirió mayor importancia a la afinidad argumentando que, dado el conocimiento mutuo del alumnado, la nacionalidad no sería tan determinante en las relaciones sociales desarrolladas: "Los problemas no se achacan a que sean españoles o marroquies. [...] Las relaciones no van a ser diferentes porque sean inmigrantes o no". De los comentarios de los estudiantes se reconoció, igualmente, la procedencia del alumnado como variable de agrupación: "No vamos a estar siempre españoles en un equipo y los marroquies en otro. Es mejor juntarnos", razonó un alumno.

Respecto a la afiliación, el maestro destacó cómo la unidad favoreció el contacto entre estudiantes que no solían interactuar en el día a día: "Les ha hecho relacionarse muchísimo más y mejor". Un estudiante lo compartió de la siguiente manera: "Yo, con (determinado) alumno, un hola y adiós por el recreo. Y ahora, con esta unidad, nos hemos hablado más". Estas interacciones se tradujeron es mayores amistades interculturales. El docente hizo especial hincapié en la calidad del clima social respecto a sus expectativas y a las características de partida del grupo-clase: "Las relaciones entre compañeros no pueden ser mejores, a pesar de lo compleja que es esta clase”, aclaró. Las ayudas prestadas entre los estudiantes parecen haber sido un síntoma de dicha mejora. Con respecto a ello, el docente destacó la existencia de un gran clima de cooperación caracterizado por el trabajo en equipo y la solidaridad intragrupal: "Cuando uno olvidaba una parte de su papel, ya estaban los compañeros para decirle lo que tenía que hacer", comentó. Los propios estudiantes así lo certificaron y destacaron la importancia de los roles a la hora de brindar ayudas: "Tus compañeros te ayudan en el error que has hecho (sic)", dijo un alumno. En estas ayudas, el docente le atribuyó un papel fundamental a la reseña de ánimos incluida en la ficha de anotación. En términos interculturales, el docente insistió en la idea de la afinidad respecto al origen cultural del alumnado: "No creo que la nacionalidad haya influido para ayudarse más o menos".

Finalmente, en cuanto a la participación del alumnado, el docente no observó diferencia alguna a razones de la procedencia de los estudiantes, sosteniendo que la implicación se dio forma generalizada: "Ha habido un esfuerzo de toda la clase", dijo. Desde su punto de vista, la unidad se desarrolló con total normalidad, sin existir discrepancias derivadas de los encuentros interculturales. Dicha normalidad fue extensible a la fase de competición en la que, aseguró, no se dio ningún tipo de sobresalto o hecho por destacar.

\section{Discusión}

El presente estudio ha tenido un doble propósito. Por un lado, examinar el impacto de una unidad didáctica de ultimate bajo las premisas del MED sobre el comportamiento y la sensibilidad interculturales de 18 estudiantes de sexto de primaria. Por otro, conocer en profundidad el desempeño de los estudiantes al actuar como entrenadores y su trascendencia en clave intercultural. 
En primer lugar, los resultados cuantitativos arrojaron mejoras significativas en la dimensión positiva de la sensibilidad intercultural para la muestra total, sin existir diferencias en razones de procedencia. Dichos resultados confirmarían la eficacia de la intervención a la hora de generar respuestas emocionales positivas hacia otros culturalmente diferentes en contextos de educación primaria. Efectos similares fueron hallados recientemente por Calderón et al. (2016) y MéndezGiménez et al. (2017), a partir de estudios MED en contextos multiculturales. En sendos trabajos la afiliación emergió como causa explicativa de mejoras en la convivencia intercultural.

En el terreno cualitativo, la tendencia observada apuntó en la misma dirección. Los cambios acaecidos pudieron ser explicados a partir de varios hechos. Por un lado, el clima de colaboración percibido, donde las ayudas intragrupales fueron señaladas como una constante a lo largo de toda la unidad, siendo congruente con los resultados encontrados por Calderón et al. (2016) y MéndezGiménez et al. (2017). Los primeros, en sintonía con lo revelado en el presente estudio, destacaron la afiliación y la confección de grupos heterogéneos como causantes del incremento en las ayudas entre iguales. Del mismo modo, la propia afiliación favoreció mayor fluidez y comprensión en los procesos de comunicación, entrando en una relación de reciprocidad respecto a las ayudas referidas. Estas conexiones entre comunicación y pertenencia al grupo han sido puestas de manifiesto recientemente en estudios con contextos similares (Martínez de Ojeda, Méndez-Giménez, y Valverde, 2016). En el presente estudio, dada la homogeneidad en cuanto al género, los agrupamientos obedecieron a criterios de procedencia cultural sirviéndose de la elevada presencia de alumnado inmigrante en el aula.

Junto con la organización social del grupo-clase, el rol de alumno-entrenador se erigió como razón de peso a la hora de explicar las diferencias observadas en la comunicación. En torno a dicho rol, fueron esgrimidos dos argumentos principales. Primero, el desarrollo de un protocolo de formación específica en el que fuesen enseñadas las claves pedagógicas para ejercer la responsabilidad de manera eficaz, este aspecto ya ha sido advertido previamente por la literatura (Farias et al., 2017; Wallhead y O'Sullivan, 2007). Un segundo factor ligado a la figura del alumno-entrenador fue la inclusión y uso de la pizarra como herramienta de andamiaje. Los trabajos previos de Farias et al. (2017) y Wallhead y O'Sullivan (2007) constataron cómo los errores de ejecución cometidos por los estudiantes eran atenuados por los alumnos-entrenadores gracias al uso de tarjetas de actividades (task cards). En el presente estudio, el uso de la pizarra ha obtenido efectos similares a aquellos propiciando, además, el uso del feedback para abordar aspectos tácticos. Tanto el docente como los estudiantes reconocieron a la pizarra como un elemento positivo y enriquecedor promotor de cultura deportiva (p.ej., surgimiento de principios tácticos) y de habilidades personales, entre las que destacan la autonomía, el liderazgo y la iniciativa.

Del mismo modo, el uso de material autoconstruido fue revelado como una decisión acertada en términos de motivación y entusiasmo. Estudios previos han evidenciado los efectos motivacionales que genera este tipo de material respecto al convencional (Méndez-Giménez, Fernández-Río, y Méndez-Alonso, 2015). En el presente estudio se reportaron muchas de las bondades asociadas a este tipo de material constatadas por la literatura (p.ej., la adaptación del material al nivel del alumnado, el desarrollo de la creatividad, o el respeto por el material propio y ajeno). En aras de confirmar los resultados hallados, futuros trabajos deberían considerar el uso de material convencional, así como la introducción de nuevos contenidos, tal y como fue sugerido desde las perspectivas docente y discente.

Por otro lado, los datos cuantitativos no reflejaron diferencias significativas en el comportamiento intercultural de los participantes, indicando que la intervención no ejerció la fuerza suficiente a efectos de modificar la conducta de los participantes. Sin embargo, considerando el plano cualitativo, las perspectivas tanto docente como discente trazaron una evolución favorable en términos de comportamiento. Se evidenciaron cambios positivos respecto al clima social de unidades previas, destacando el desarrollo de un ambiente caracterizado por el respeto mutuo y el disfrute. También se recalcó la capacidad de los estudiantes para superar sus primeros desencuentros (p.ej.: elección de roles, composición de los equipos, etc.). Estas consideraciones, junto a otras manifestadas a lo largo de la unidad, confirmarían el potencial del MED para paliar tanto situaciones conflictivas como conductas disruptivas (Calderón et al., 2016; Martínez de Ojeda et al., 2016). Otro aspecto por destacar, señalado al inicio de esta discusión, ha sido la ausencia de significatividad en función de la 
procedencia del alumnado. El análisis de este estudio es concordante con lo dispuesto por Calderón et al. (2016) quienes diagnosticaron el hecho referido como rasgo positivo de su intervención al concluir que el proceso de integración resultante se había dado de forma genérica a todos los estudiantes, más allá de su origen cultural.

Finalmente, este estudio ha contado con una serie de limitaciones. En primer lugar, el propio diseño de investigación, por dos razones: la carencia de grupo-control y el reducido tamaño de la muestra. En segundo lugar, cabe destacar la ausencia de participantes de sexo femenino, con la dificultad que comporta a la hora de extrapolar los resultados y replicar la intervención en escenarios similares. Por último, es necesario considerar los bajos índices de fiabilidad obtenidos en varias de las sub-escalas administradas a los participantes. Futuros trabajos deberían considerar la pertinencia de estas y optar por aquellas que mejor se ajusten a las características de los sujetos.

\section{Conclusiones}

A tenor de lo expuesto, los resultados encontrados parecen indicar que las intervenciones realizadas bajo las premisas del MED favorecen la integración de los estudiantes independientemente de sus características individuales, siendo la procedencia del alumnado una de ellas. No obstante, la ausencia de significatividad respecto al comportamiento intercultural motivada, en gran parte, por la duración de la intervención, sugiere el diseño de estudios longitudinales en vistas al futuro.

Los resultados del presente estudio aportan datos de interés práctico. En primer lugar, se ha constatado la necesidad de atender a la composición de los equipos establecidos a efectos de auspiciar espacios de reciprocidad e intercambio. No obstante, cabe destacar que la afiliación se ha mostrado como condición necesaria pero no suficiente si lo que se pretende es brindar al alumnado oportunidades eficaces para el desarrollo de su competencia intercultural. Considerando aspectos más globales del aprendizaje, hubiese resultado insuficiente saldar el proceder pedagógico con decisiones ceñidas exclusivamente a la organización social del aula.

Así pues, otras de las implicaciones didácticas que se derivan de esta intervención abogan por el uso de material variado. El uso de la pizarra se ha presentado como técnica eficaz de andamiaje capaz de facilitar y enriquecer el proceso de comunicación. Su inclusión ha reportado beneficios tanto a los estudiantes que ejercieron el rol de entrenador como a sus compañeros en calidad de receptores. Futuras intervenciones deberían ir dirigidas a explorar en mayor profundidad el uso de esta herramienta a partir de estudios prolongados y en contextos diferentes, como puede ser la etapa secundaria o escenarios con bajo porcentaje de alumnado inmigrante. Respecto al rol de alumnoentrenador, cabe insistir en la necesidad de desarrollar procesos de formación específica orientados a brindar un aprendizaje suficiente de las labores y funciones que comporta dicha responsabilidad. Para ello, se ha de ser consciente de que ciertos procesos instructivos requieren un tiempo determinado para ser aprendidos (Farias et al., 2017), pudiendo ser más extensos y sensibles en contextos multiculturales debido, entre otras razones, a posibles barreras idiomáticas y a los estilos de aprendizaje que porta consigo el alumnado.

\section{Referencias}

Aguado, T. \& Ballesteros, B. (2015). Investigando la escuela intercultural. Experiencias y resultados del Grupo INTER. Capítulo 7. En A. Escarbajal (Ed.). Comunidades interculturales y democráticas (pp. 99-112). Madrid: Narcea.

Arslan, Y., Gunçavdi, G., \& Polat, S. (2014). The impact of peace education programme at university on university students' intercultural sensitivity. Procedia - Social and Behavioral Sciences, 174, 2301-2307. DOI: $10.1016 /$ j.sbspro.2015.01.891.

Besalú, X. (2002). Diversidad cultural y educación. Madrid: Síntesis.

Boyd, C. (2001). Combining qualitative and quantitative approaches. En P. L. Munhall (Ed.), Nursing Research: A Qualitative Perspective. Third edition, 579-598. Sudbury, MA: Jones and Bartlett.

Calderón, A., Martínez de Ojeda, D., Valverde, J. J., \& Méndez-Giménez, A. (2016). "Ahora nos ayudamos más”: Docencia compartida y clima social de aula. Experiencia con el modelo de Educación Deportiva. 
RICYDE. Revista Internacional de Ciencias del Deporte, 44(12), 121-136. DOI: 10.5232/ricyde2016.04403.

Campbell, D. T., \& Stanley, J. C. (1963). Experimental and quasiexperimental designs for research and teaching. En N. L. Gage (Ed.), Handbook of research on teaching. Chicago, IL: Rand McNally.

Chen, G.M. \& Starosta, W. J. (2000). The development and validation of the Intercultural Sensitivity Scale. Human Communication, 3(1), 2-14.

Cohen, J. (1988). The concepts of power analysis. En J. Cohen (Ed.). Statistical Power Analysis for the Behavioral Sciences, 1-18. Hillsdale, NJ: Lawrence Erlbaum Associates.

Cohen, L. \& Manion, L. (2002). Métodos de investigación educativa. Madrid: La Muralla.

Cuevas, R., García-López, L. M., \& Contreras, O. (2015). Influencia del modelo de Educación Deportiva en las necesidades psicológicas básicas. Cuadernos de Psicología del Deporte, 15(2), 155-162. DOI: $10.4321 / \mathrm{S} 1578-84232015000200017$

Derri, V., Kellis, I., Vernadakis, N., Albanidis, E. \& Kioumourtzoglou, E. (2014). The Effect of an Intercultural Physical Education Program in Comparison to the Typical One on Students' Social Skills Learning. Journal Human Sport Exercise, 9(1), 91-102.

Dyson, B., Griffin, L.L. \& Hastie, P. A. (2004). Sport education, tactical games and cooperative learning: Theorical and pedagogical considerations. Quest, 56, 226-240.

Ennis, C. D. \& Chen, S. (2012). Interviews and focus groups. En K. Armour \& D. Macdonald (Ed.), Research Methods in Physical Eduction and Youth Sport. (pp. 217-236). New York: Routledge.

Erickson, F. (1989). Métodos Cualitativos de Investigación Sobre la Enseñanza. En Wittrock, Merlin C. (Ed.). La Investigación de la Enseñanza, II - Métodos Cualitativos y de Observación (pp. 125-301). Barcelona: Ediciones Paidós.

Evangelio, C., González-Víllora, S., Serra-Olivares, J., \& Pastor-Vicedo, J. C. (2016). El Modelo de Educación Deportiva en España: Una revisión del estado de la cuestión y prospectiva. Cuadernos de Psicología Del Deporte, 16(1), 307-324.

Farias, C., Hastie, P. A. \& Mesquita, I. (2017). Scaffolding student-coaches' instructional leadership toward student-centred peer interactions. A yearlong action-research intervention in sport education. European Physical Education Review. Prepublicado: 13-1-2017. DOI: 10.1177/1356336X16687303.

Fritz, C. O., Morris, P. E., \& Richler, J. J. (2012). Effect size estimates: current use, calculations, and interpretation. Journal of Experimental Psychology: General, 141(1), 2-18.

García, J. A. \& Goenechea, C. (2009). Educación intercultural. Análisis de la situación y propuestas de mejora. Wolters Kluwer. España: Madrid.

García-López, L. M., \& Gutierrez, D. (2016). Aprendiendo a enseñar deporte. Modelos de enseñanza comprensiva y educación deportiva. Barcelona: INDE.

Gutiérrez-Díaz, D., García-López, L. M., Chaparro, R., \& Fernández Sánchez, A. J. (2014). Aplicación del modelo de educación deportiva en segundo de Educación Primaria. Percepciones del alumnado y el profesorado. Cuadernos de Psicología del Deporte, 14(2), 131-144.

Gutiérrez-Díaz, D., García-López, L. M., Hastie, P. A., \& Calderón, A. (2013). The responses of Spanish students' to participation in seasons of sport education. The Global Journal of Health and Physical Education Pedagogy, 2(2), 111-127.

Hambleton, R. K., Merenda, P., \& Spielberger, C. (Eds.) (2005). Adapting educational and psychological tests for cross-cultural assessment. Mahwah, NJ: Lawrence Erlbuam Associates.

Hastie, P. A., Buchanan, A., Wadsworth, D., \& Sluder, B. (2009). The Impact of an Obstacle Course Sport Education Season on Students' Aerobic Fitness Levels. Research Quarterly for Exercise and Sport, 80(4), 788-791.

Hastie, P. A., Martínez de Ojeda, D., \& Calderón, A. (2011). A review of research on Sport Education: 2004 to the present. Physical Education $y$ Sport Pedagogy, 16(2), 103-132. DOI: 10.1080/17408989.2010.535202

Hastie, P. A. \& Sinelnikov, O. A. (2006). Russian students' participation in and perceptions of a season of Sport Education. European Physical Education Review, 12, 131-150. DOI: 10.1177/1356336X06065166

Jiménez, R. \& Goenechea, C. (2014). Educación para una ciudadanía intercultural. Madrid: Síntesis. 
Johnson, G. M. \& Miller, P. J. (1993). Field theory applied to classrooms: toward a bidirectional paradigm of teacher-student relationships. The Journal of Classroom Interaction, 28(2), 15-21.

Jordán, J. A. (1999). ¿Qué educación intercultural para nuestra escuela? http://www.aulaintercultural.org/IMG/pdf/jordan_escuela.pdf

Kellis, I., Vernadakis, N., Albanidis, E., Derri, V., \& Kourtesses T. (2010). The development of a student's behaviors' self-evaluation scale (SBSS) in multicultural physical education class settings. Educational Research and Review, 5(11), 637-645.

Kline, P. (1998). The new psychometrics: Science, psychology and measurement. New York, EEUU: Routledge.

Kouli, O. \& Papaioannou, A. G. (2009). Ethnic/cultural identity salience, achievement goals and motivational climate in multicultural physical education classes. Psychology of Sport and Exercise, 10, 45-51. DOI: 10.1016/j.psychsport.2008.06.001.

Lin, T.-C., Hsu, Y.-S., Lin, S.-S., Changlai, M.-L., Yang, K.-Y., \& Lai, T.-L. (2012). A Review of Empirical Evidence on Scaffolding for Science Education. International journal of Science and Mathematics Education, 10, 437-455. DOI: 10.1007/s10763-011- 9322-z

López, O. \& Hederich, C. (2010). Efecto de un andamiaje para facilitar el aprendizaje autorregulado en ambientes hipermedia. Revista Colombiana de Educación, 58, 14-39. DOI: 10.17227/01203916.631.

Martínez de Ojeda, D., Méndez-Giménez, A., \& Valverde, J. J. (2016). Efectos del modelo Educación Deportiva en el clima social del aula, la competencia percibida y la intención de ser físicamente activo: un estudio prolongado en primaria. Sport TK, 5(2), 153-165.

Massot, I., Dorio, I., \& Sabariego, M. (2004). Estrategias de recogida y análisis de la información. En R. Bisquerra (Coord.). Metodología de investigación educativa (pp. 329-366). Barcelona: La Muralla.

Méndez-Giménez, A., Fernández-Río, J., \& Méndez-Alonso, D. (2015). Modelo de Educación Deportiva versus Modelo Tradicional: Efectos en la motivación y deportividad. Revista Internacional de Medicina y Ciencias de la Actividad Física y el Deporte, 15(59), 449-466. DOI: 10.15366/rimcafd2015.59.004.

Méndez-Giménez, A., Puente-Maxera, F., \& Martínez de Ojeda, D. (2017). Efectos de una unidad didáctica de mimo basada en el modelo de Educación Deportiva sobre la interculturalidad. Sport TK, 6(2), 89-100.

Metzler, M. W. (2005). Instructional Models for Physical Education. (2 ${ }^{\mathrm{a}}$ ed.) Arizona: Holcomb Hathaway, Publishers, Inc.

Ruokonen, I. \& Kairavuori, S. (2012). Intercultural Sensitivity of the finnish ninth graders. Procedia - Social and Behavioral Sciences, 45, 32-40. DOI: 10.1016/j.sbspro.2012.06.540.

Sanhueza, S. V. (2010). Sensibilidad intercultural: un estudio exploratorio con alumnado de educación primaria y secundaria en la provincial de Alicante. Tesis doctoral. Universidad de Alicante.

Sanhueza, S. V. \& Cardona, M. C. (2008). Escala de Sensibilidad Intercultural para Alumnado de Primaria y Secundaria (documento inédito). Alicante: Universidad de Alicante.

Sanhueza, S. V. \& Cardona, M. C. (2009). Evaluación de la sensibilidad intercultural en alumnado de educación primaria escolarizado en aulas culturalmente diversas. Revista de Investigación Educativa, 27(1), 247262.

Siedentop, D., Hastie, P. A., \& H. van der Mars (2011). Complete Guide to Sport Education, Second Edition. Champaign, IL: Human Kinetics.

Sierra-Arizmendiarrieta, B., Méndez-Giménez, A., \& Mañana-Rodríguez, J. (2013). La programación por competencias básicas: hacia un cambio metodológico interdisciplinar. Revista Complutense de Educación, 24(1), 165-184. DOI: http://dx.doi.org/10.5209/rev_RCED.2013.v24.n1.41196.

Wallhead, T. \& O'Sullivan, M. (2005). Sport education: Physical education for the new millennium? Physical Education y Sport Pedagogy, 10(2), 181-210. DOI: 10.1080/17408980500105098.

Wallhead, T. \& O'Sullivan, M. (2007). A didactic analysis of content development during the peer teaching tasks of a sport education season. Physical Education and Sport Pedagogy, 12, 225-243. DOI: $10.1080 / 17408980701610177$.

Ward, P. \& Lee, M. (2005). Peer-assisted learning in physical education: A review of theory and research. Journal of Teaching in Physical Education, 24, 205-225.

Weimer, M. (2002). Learner-Centred Teaching: Five Key Changes to Practice. San Francisco: Jossey-Bass.

Wood, D., Bruner, J. S., \& Ross, G. (1976). The Role of Tutoring in Problem Solving. Journal of Child Psychology and Psychiatry, 17, 89-100. 